\title{
Role of Histogram Modification Functions EQH, LOG, POLY, Linear Equations for CBIR Based on 64 Bins Approach
}

\author{
Dr. H. B. Kekre and Kavita Sonawane \\ Department of Computer Engineering, NMIMS University, Vileparle, Mumbai, India \\ Ph.D. Research Scholar, NMIMS University, Mumbai \\ hbkekre@yahoo.com, kavitavinaysonawane@gmail.com
}

\begin{abstract}
This paper describes the use of histogram modification functions to improve the retrieval efficiency of the content-based image retrieval system based on bins approach. Four different functions explored in this paper used as histogram specification to modify the histograms are; histogram equalization (EQH), polynomial function (POLY), linear equations (LinearEQ1,2,3) and logarithmic function (LOG). Modified histograms are partitioned into 4 parts using centre of gravity (CG) to form 64 bins from 256 bins of $R, G$ and $B$ histograms. Bins holding the count of pixels falling in particular range of modified intensities divided into four parts using centre of gravity. Statistical properties are computed for the intensities possessed by the pixels counted into these 64 bins. These properties are representing the type of feature vector in the form of first four moments namely mean, standard deviation, skewness and kurtosis. All moments are computed for three-color intensities $R, G$ and B separately. Based on color and moment different types of feature vector databases are prepared. Comparisons of query feature vector with feature vectors of database images is carried out by means of three similarity measures namely Euclidean distance (ED), absolute distance $(A D)$ and cosine correlation distance $(C D)$. Role of each modification function along with all types of feature vectors is evaluated and compared and presented using three parameters; Precision Recall Cross over Point (PRCP), Longest String(LS), Length of String to Retrieve all Relevant (LSRR).
\end{abstract}

Keywords: Bins, histogram modification, Mean, Standard Deviation, Skewness, Kurtosis, PRCP, LS, LSRR

\section{Introduction}

Content based image retrieval methods always motivates us to have better results rather than just being stratified at good results. This area has huge scope for researchers to work out and recommend the simple and effective techniques for large number of retrieval of relevant images based on image contents. One reason for this is definitely the drawbacks of text based image retrieval methods. It is also expected by CBIR users to have simple but effective techniques to improve the retrieval efficiency. CBIR methods are dealing with various image contents, which include mainly three contents color, texture, and shape which are subjective to human visibility. These contents can be extracted and represented in various ways to form the feature vector representing that image. Various techniques are invented in spatial and frequency domain [1-7]. Various approaches using color contents because color information is invariant to scaling and rotation which will always remain constant for multiple copies of same image may be present in different angles [8-10]. Texture feature is indirect measure of the image contents defining the quality or textural properties of the image. Perceptually 
looking same images may have different texture feature. Use of only one content feature to represent or to compare that image with other images is not feasible or enough. It requires the combination of these contents to form a strong feature vector representing that image effectively. In this work we are mainly concentrating on the color content of the image and indirectly on the texture features. Color information is being processed in terms of color histograms of the image and textural are computed by means of statistical properties of the pixels intensities [11-14]. This paper explores the CBIR techniques based on histogram modification and histogram partitioning for bins approach. Many approaches are based on histogram bins. Some are using local histogram, some are using global histogram. In local histogram approach they are dividing the image into multiple blocks and for each block a histogram will be obtained [15-19]. Refined histograms, shifted histograms are also used for feature vector generation in few CBIR systems. All these approaches if observed it has been found that for indexing purpose the comparisons carried out using all 256 bins of histogram as it is. It increases the computational complexity, as the size of the feature vector is big. Few of them are randomly selecting the bins to be used as feature of for the image comparisons. It may miss some significant content of the image that may have good impact on the results [2023]. This work we have tried to overcome the problem of increasing computational complexity by reducing the size of the feature vector. Instead of using the 256 bin of histogram the feature vector size is brought to 64 bins only. This dimensionality reduction is achieved by the bins formation approach proposed in this paper. This process is based on two different methods of partitioning the histogram that is linear partitioning and centre of gravity based partitioning. The work has been carried out using both methods but only better results obtained for CG partitioning are presented here. Image indexing and retrieval is also an important phase of any CBIR system. It includes the query image comparisons with database images. Many ways are available to show how to enter the query image into the system. Various similarity measures can be used as per literature to carry out this comparison it includes the Minkowiski distance, Manhattan distance, cosine correlation distance, quadratic distance etc [24-26]. We are making use of three similarity measures for the same purpose that are, Euclidean distance, absolute distance and cosine correlation distance. Image is similar to query or not is determined based on distance, the minimum is the distance indicates the similarity with query. System generates the retrieval set of images, which includes relevant and irrelevant images. Ideal system should generate retrieval set having only relevant images. However, this is very difficult get such situation. It is always better to analyze the system's performance to determine the idealness of it. To do this various researcher are using conventional measures precision and recall as performance evaluation parameters [27-29]. We are using three parameters to analyze the system's performance that are; Precision Recall Cross over Point (PRCP), Longest String (LS), LSRR (Length of String to Retrieve all Relevant). Further presentation of the paper is as follows: Section 2 describes the Histogram and histogram modification functions. Section 3 explains the Histogram partitioning and bins formation-using CG. Section 4 elaborates the feature vector extraction process. Section 5 is focusing on application of similarity measures for indexing and retrieval and defines the performance evaluation parameters. Section 6 presents the experimental results and discussion followed by conclusion in Section 7.

\section{Histogram and Histogram Modifications}

Histogram is the simple plot, indicating the number of occurrences of each grey level. It can be defined as graphical representation of the tonal distribution in a digital image. This graph is nothing but the representation of pixel distribution as a function of tonal variation. 
Color histograms will give us the clear idea about the color distribution in terms of the number of pixels of that particular color intensity. Color histograms can be built to any type of color space. Here we are working with the R, G and B color space. We read the image, separate it into R, G and B planes and then compute the histograms of each plane. Histograms are invariant to scaling and rotation transformations and can be used effectively for feature extraction and representation in CBIR [29-31]. Following Figure 1 shows the image with R, $\mathrm{G}$ and $\mathrm{B}$ planes along with their respective histograms in Figure 2.

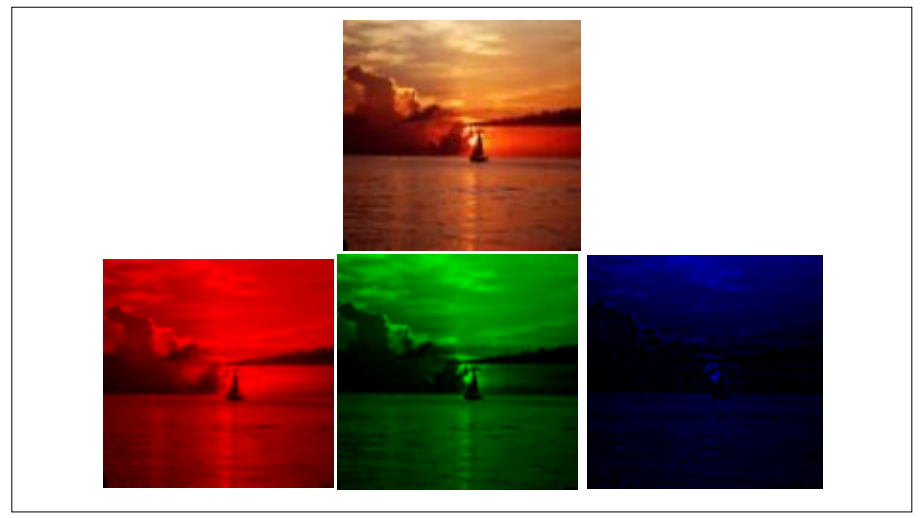

Figure1. Sunset Image with R, G and B Planes Separated

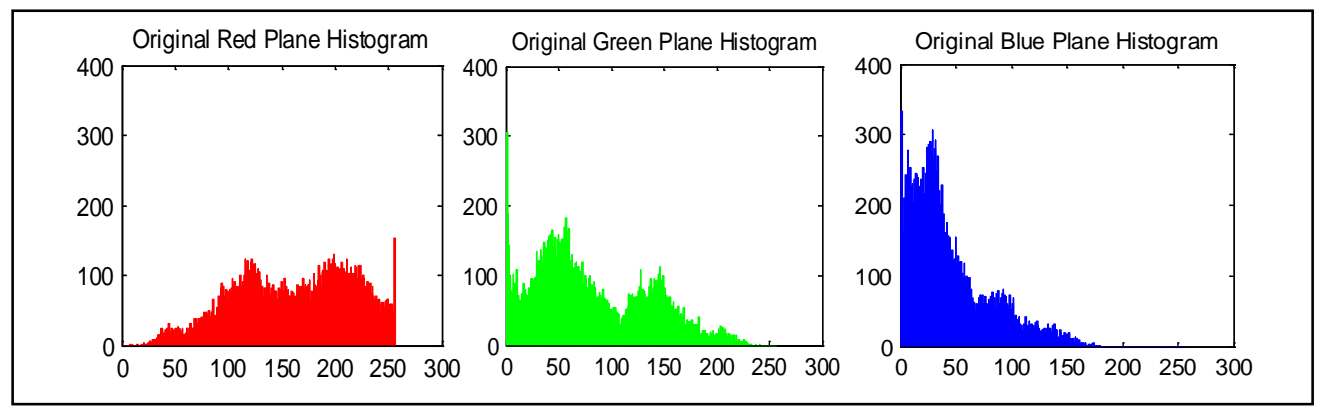

Figure 2. R, G and B Plane Histograms of Sunset Image

\subsection{Histogram Modifications}

Histogram modifications are the histogram modelling techniques provide sophisticated method for modifying the dynamic range and contrast of an image. This can be done by modifying the image such that its intensity histogram will have desired shape. We are trying to modify the images in such a way that small details can be captured and the image contents can be used efficiently. The modification functions used are defined as follows: EQH, LOG, POLY, and Linear Equation1, 2, \&3. Each one of them along with its impact on red plane of an image is explained below.

2.1.1. EQH: Histogram Equalization: This is widely used histogram modeling technique that tries to distribute all image pixels to cover entire range of available intensities. It employs a monotonic, non linear mapping which re-assigns the intensity values of pixels such that the output image contains uniform distribution of intensities. Histogram will take nearly flat shape because of digitization of images. 


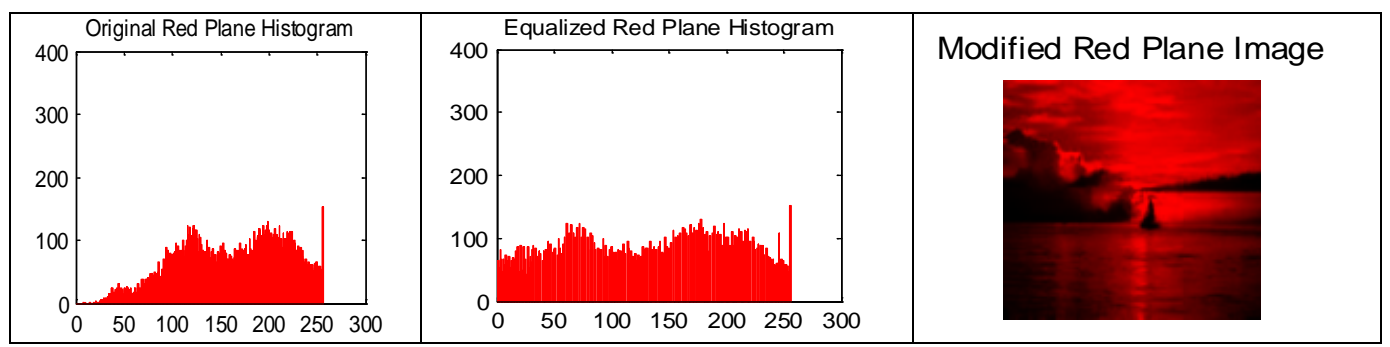

Figure 3. Sunset Red Plane: From Left Histograms Original, Equalized and Equalized Image

2.1.2. LOG: Logarithmic Functions: Logarithmic Transformations can be used to brighten the intensities of an image. It is used to expand the values of dark pixels in an image while compressing the higher values. It maps the narrow range of low input grey level values into wider range of output values.

$$
y=C * \log _{10}(x+1) \quad \text { where } C=\frac{1}{\log _{10}(2)}=1.44
$$

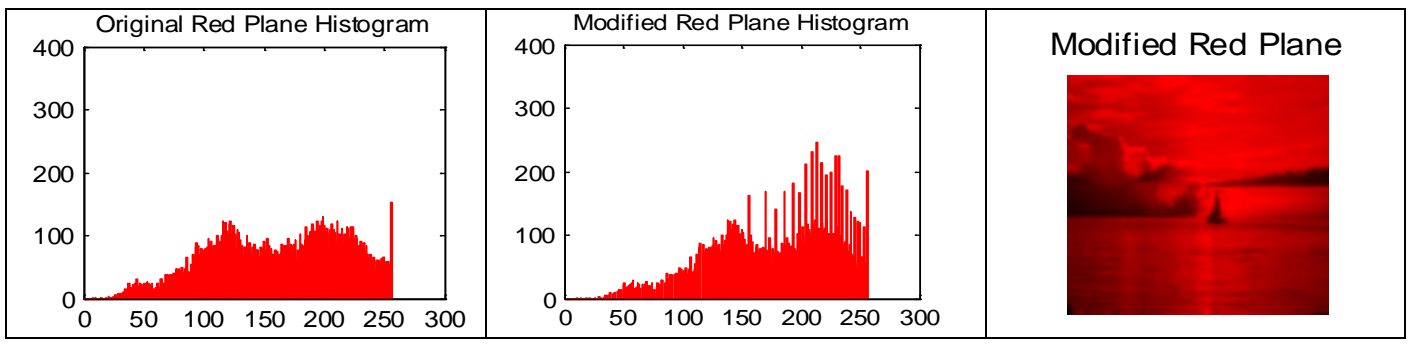

Figure 4. Sunset Red Plane: From Left Histograms Original, LOG Modified Histogram and Image

2.1.3. POLY: Polynomial function: It is a simple polynomial expression which actually pushes the original histogram intensities from lower to upper side. It brings positive change in the low key images.

$$
y=2 x-x^{2}
$$

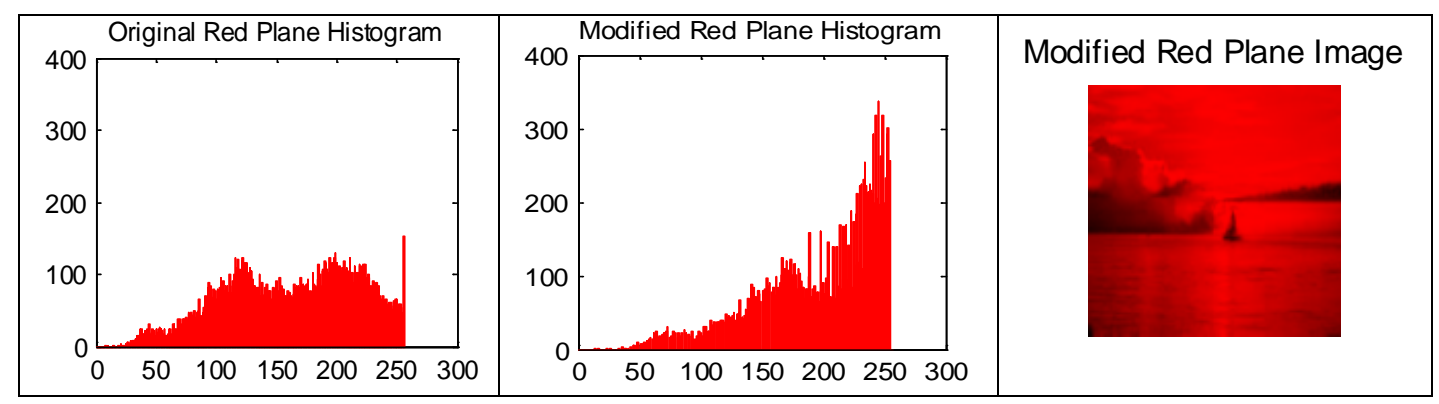

Figure 5. Sunset Red Plane: From Left Histograms Original, POLY Modified Histogram and Image 
2.1.4. LINEQ: LinearEquation1, 2 and 3: The proposed linear equations are also designed to push the intensities from low to high level linearly. The linear equations designed in parts as explained in our paper [15].

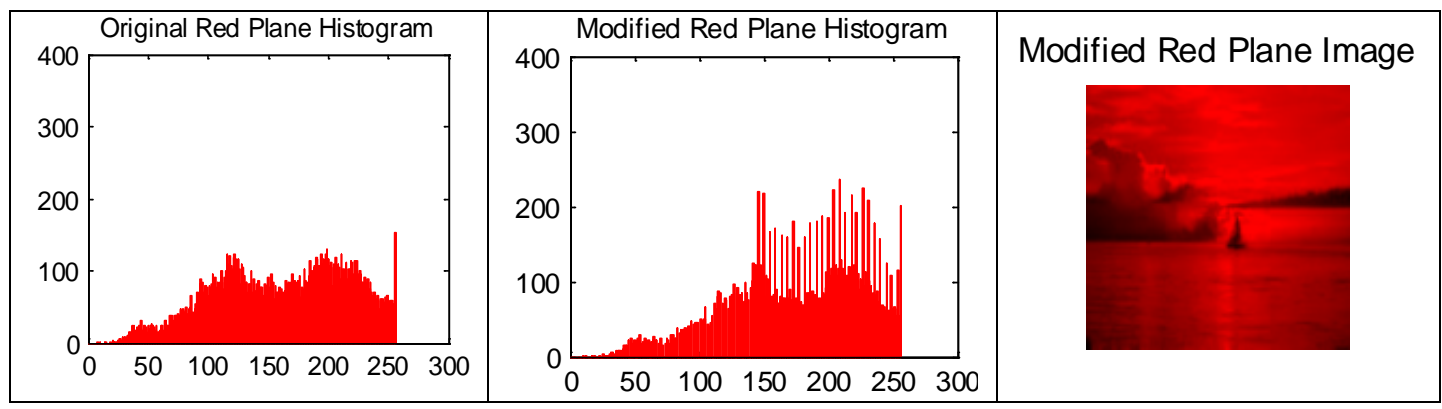

Figure 6. Sunset Red Plane: From Left Histograms Original, LINEQ Modified Histogram and Image

In this work the role of each modification function implemented and applied over the R, G and B histograms of an image. Purpose of using these modifications is very straight-forward. We are trying to enhance the image so that it can be used effectively in the comparison process. Through this point of view, we have analyzed and evaluated the performance of each modification function for CBIR.

\section{Histogram Partitioning and Bins Formation}

As the proposed CBIR techniques are based on bins approach; let us understand how this bins formation process takes place. This process starts with the partitioning of histograms of $\mathrm{R}, \mathrm{G}$ and $\mathrm{B}$ planes of an image. Partitioning is used to reduce the size of the feature vector in terms of number of bins. Many CBIR systems are based on histogram. They are using the histogram bins i.e., giving the count of pixels of each intensity level as feature vector. These bins are either selected; all bins as feature vector or linearly or based on some grouping logic [29-31]. In this work a novel idea for partitioning is introduced where the R, G and B histograms partitioned into 4 parts using centre of gravity. We have also worked out linear partitioning before CG. It is observed that CG partitioning gives far better results compared to linear partitioning. That is why in this paper, we are using only CG partitioning.

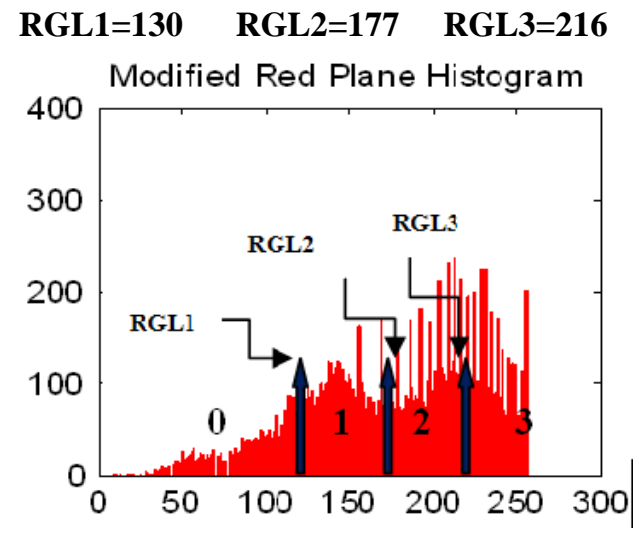

Figure 7. CG Partitioning over LOG modified Red Histogram 
CG Partitioning: According to this, first we partitioned the histogram into two parts by computing CG where two parts will have same moments around it. Further same logic is used to divide these two parts into four. Figure 7 Shows the CG partitioning. Each of these four parts are identified with the part ids 0,1, 2 and 3. Now the bins formation takes place as follows:

Let's pick up the pixel $P(i, j)$ from the image under feature extraction process and checks its R, G and B intensities Rp(i, j), Gp(i, j), and Bp(i, j). Each if these values will get a part id assigned to them on the basis of which part of respective histogram they fall. e.g., we check pixel P(i, j)’s R, G and B intensities and the following conditions are found

If $\mathrm{Rp}(\mathrm{i}, \mathrm{j})$, falls in partition ' 0 ' of red plane histogram

If $\mathrm{Gp}(\mathrm{i}, \mathrm{j})$, falls in partition ' 1 ' of green plane histogram

If $\mathrm{Bp}(\mathrm{i}, \mathrm{j})$, falls in partition ' 3 ' of blue plane histogram

Then pixel $P(i, j)$ will get flag '013' which is the destination bin address 'Bin7' for $P(i, j)$ to be counted. This is the way the addresses are determined for each pixel to be counted as their destination bin. Three planes divided into 4 parts generate 64 bins range from 000 to 333. All the pixels of the image under feature extraction process will be distributed into these 64 bins based on intensity range they are falling in.

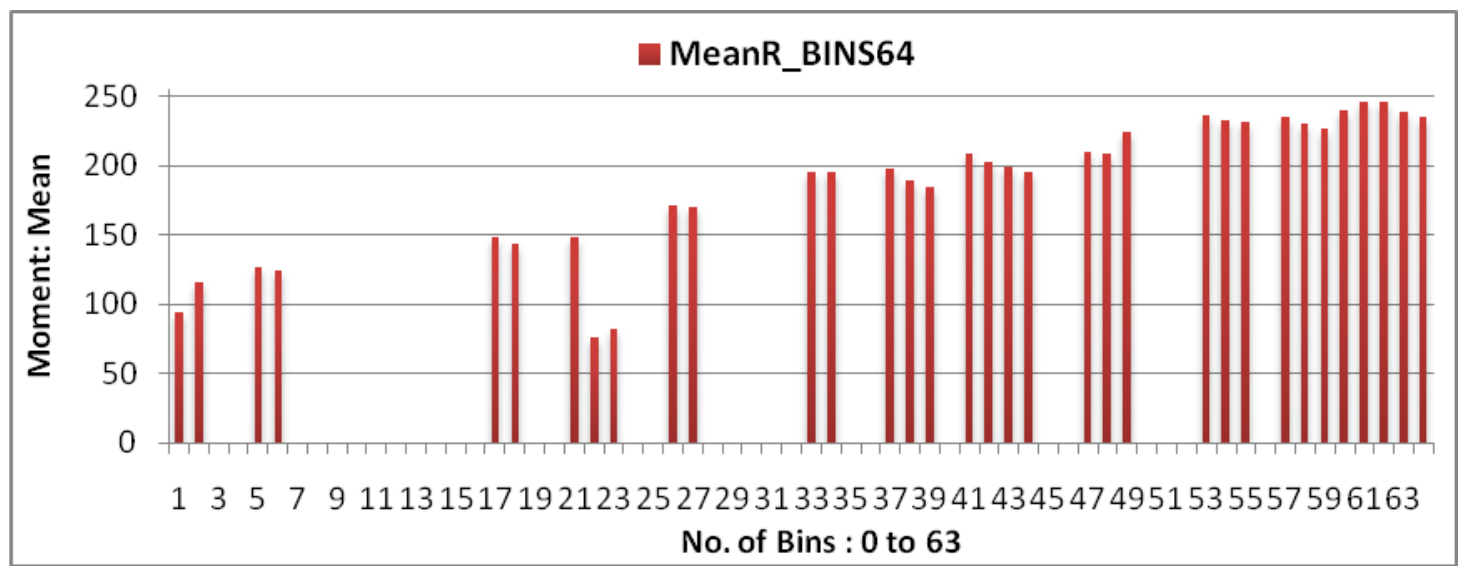

Figure8. Sample 64 Bins for Mean of Red Intensities for the Pixels Counted into
each of the 64 Bins

Sample of 64 bins are shown in Figure 8. It shows the Mean of pixel's red intensities into each of the 64 bins. Few bins are blank fields as the count of pixels into those bins is zero. Instead of using the 256 bins of each R, G and B histogram as it is, we have reduced the feature vector size to just 64 bins. It reduces the computational complexity.

\section{Feature Vector Extraction}

Feature extraction is the core phase of all CBIR systems which actually deals with the image contents to be extracted, processed and to be presented in the form of feature vector. Block diagram in Figure 9 indicates the neat and simple view of the feature extraction process.

Feature extraction shown in Figure 9, starts with separation of R, G and B planes of image under feature extraction process. Then we compute the histograms for them which are Modified using 4 different modification functions namely EQH, POLY, Linear EQ and LOG 
as are explained in Section 3. After modifying the R, G and B histograms using desired function, they will be divided into 4 equal parts by means of centre of gravity (CG). These parts are identified by ids $0,1,2$ and 3. Three planes each divided into 4 parts generate the 64 bin addresses.

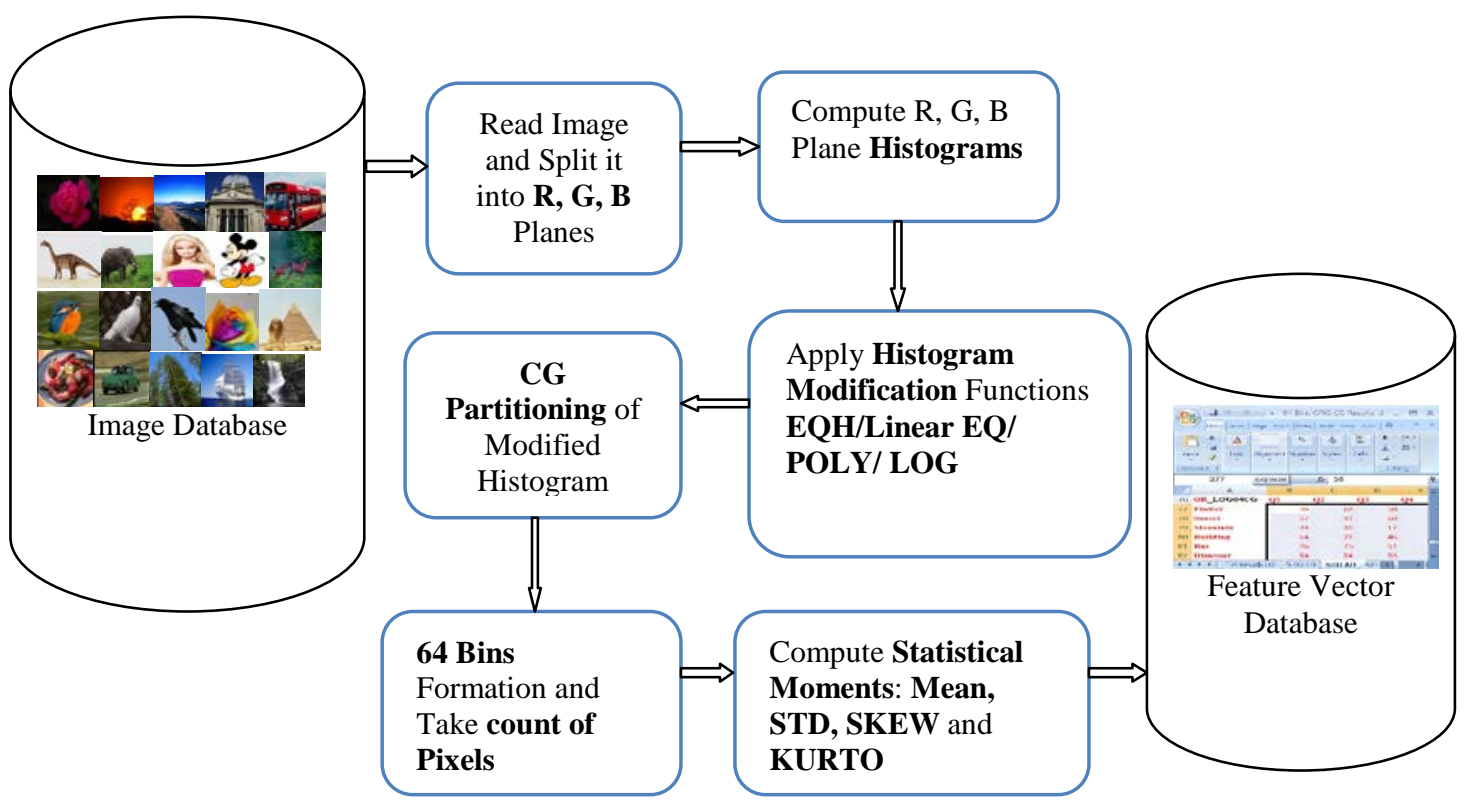

Figure 9. Feature Extraction Process of Proposed CBIR Techniques

Initially these 64 bins are holding the count of pixels falling in particular range of $\mathrm{R}, \mathrm{G}$ and B intensities of respective R, G and B histograms. Color histograms can potentially be identical for two images with different objects if they are having the same color information. That is why instead of considering only count of pixels as a feature vector; we are computing the statistical properties of that data to increase discriminating strength of the feature vector. The statistical properties computed are the first four moments namely mean, standard deviation, skewness and kurtosis. This way we can notice that along with the color feature, texture feature is also being considered in this work.

The moments are calculated separately for each color. Feature vector based on different color and different moment treated separately and stored in separate feature vector database. This way for one image we can have R, G and B feature each with four moments for original and histograms modified using four different functions EQH, POLY, Linear Equations and LOG. In all, one image can be represented using 60 different feature vectors. This process is repeated for all the database images and 60 different feature vector databases are prepared as pre-processing work. This is the overall workflow of the block diagram shown in Figure 9.

\section{Indexing, Retrieval and Performance Evaluation}

This the last phase of CBIR systems where the query image will be compared with database images by means of calculating the distance between their feature vectors. These distances will decide the similarity between them. Images at far distance from query are treated as irrelevant images whereas images at minimum distance are considered to be relevant images. This is what the indexing and retrieval process. In many CBIR systems threshold is determined for the retrieval and then performance of the system will be evaluated 
using the most commonly used parameters, precision and recall. Similarity measures and performance evaluation parameters used in this paper are explained below.

\subsection{Application of Similarity Measures and Retrieval}

Once the feature vector databases are prepared for all database images, system is ready to face the query image. Whenever query image enters in the system, all feature vectors (60) are calculated for the same. These feature vectors are then compared with the respective feature vector of all database images. This comparison is carried out by means of three similarity measures namely Euclidean distance (ED), Absolute distance (AD) and Cosine correlation distance (CD). These similarity measures are defined in equations 4, 5 and 6 respectively. Distance between the query image and all database images is computed and sorted in ascending order. Images at minimum distance from query can be selected as similar images.

Euclidean Distance :

Absolute Distance:

Cosine correlation distance (CD)

$$
D_{Q I}=\sqrt{\sum_{i=1}^{n} \mid\left(F Q_{i}-F I_{i}\right)^{2}}
$$

$$
D_{Q I}=\sum_{1}^{n}\left|\left(F Q_{I}-F I_{I}\right)\right|
$$

$$
\frac{\langle D(n) \mid Q(n)\rangle}{\sqrt{\langle D(n) \mid D(n)\rangle\langle Q(n) \mid Q(n)\rangle]}}
$$

where $\mathrm{D}(\mathrm{n})$ and $\mathrm{Q}(\mathrm{n})$ are Database and Query feature Vectors resp.

\subsection{Performance Evaluation Parameters: PRCP, LS and LSRR}

PRCP: Precision Recall Cross over Point: After computing the distance between the query and database images the retrieval process takes place. After computing the distance they are sorted in ascending order. According to the conventional methods a threshold will be determined on trial and error basis. Retrieval set will be obtained and precision and recall for the same will be calculated. Like other CBIR systems, instead of determining the threshold for collecting the relevant images we have used little modified logic. Parameters precision and recall are defined as follows in equations 7 and 8 respectively.

$$
\begin{aligned}
& \text { Precision }=\frac{\text { Relevant Retreived Images }}{\text { All Retrieved Images }} \\
& \text { Recall }=\frac{\text { Relevant Retreived Images }}{\text { All Relevant In Database }}
\end{aligned}
$$

In this work we are taking the cross over point of precision and recall, where precision and recall both are same. To implement this logic the set of retrieval is set of first 100 images collected with respect to first 100 images according to sorted distances (obtained for 2000 database images). PRCP is nothing but the collection of relevant images from these first 100 images. As we have 100 images of each category in the database; it becomes a crossover 
point where the precision and recall will be same. Precision is the measure of accuracy whereas recall is measure of completeness. Cross over point of both of these parameters is interpreting the idealness of the system, if it (PRCP) is =1or may indicate the worst performance of the system if it is $=0$;

LS: Longest String: This parameter interprets the strength of the system in terms of continuous string of relevant retrieved images from the large size database. As discussed above when we take the first 100 images into consideration according to the sorted distances. In LS we are searching for the longest continuous string of relevant images from sorted database images. Maximum LS proves the best system performance.

LSRR: Length of String to Retrieve all Relevant. This parameter measures the length of the traversal of sorted distances (from 1 to 2000 in this work) to collect all relevant images. Retrieving all relevant is nothing but making recall 1 . The early we reach to recall $=1$; indicates the best performance of the system. Means LSRR for the ideal CBIR system should be as low as possible.

\section{Experimental Results and Discussion}

The experimentation of proposed approaches based on histogram and its modifications along with the bins approach is carried out over database of 2000 BMP images. This image database is prepared of 20 different categories which includes few from Wang database [32]. The 20 different classes we used are Flower, Sunset, Mountains, Bus, Dinosaur, Elephant, Barbie, Mickey, Horse, Kingfisher, Dove, Crow, Rainbow rows, Pyramids, Plate, Car, Trees, Ship and Waterfall. Each class has 100 images of its own category. As explanation of feature extraction process in section $\mathrm{V}$, the same process is applied to these all $2000 \mathrm{BMP}$ images in the database and feature vector databases for them are prepared as pre processing work. Based on different types of feature vector with respect to the histogram modification functions, different colors and different moments with respect to CG partitioning, we have prepared total 60 feature vector databases. We actually have worked out all with Linear partitioning too; but the results of CG with all other factors are far better than LP and that is why we explored the proposed approaches only with respect to CG partitioning.

\subsection{Query by Example}

Once the feature vector databases are ready a query can be fired to the system. There are various ways through which the query can be inputted to the system. However we are entering an image as query to the system. Query can be selected from the database or outside the database as an example image and that is why it is called "query by example approach". To test the response of CBIR system based on proposed approaches we prepared set of 200 query images. It includes 10 images selected randomly from each of the 20 classes.

\subsection{Results and Discussion:}

Whenever the query enters into the system, the feature vector for the same will be extracted and represented in all different types as processed for all the database images. This feature vector will be then compared with the prepared feature vector databases. Comparison is carried out using the three similarity measures $\mathrm{ED}, \mathrm{AD}$ and $\mathrm{CD}$. Retrieval results after calculating the distance are presented and evaluated in terms three performance evaluation parameters namely PRCP, LS and LSRR. All the proposed approaches are executed using same set of 200 query images. Result of each individual query executed is stored separately. Because of space limitations directly total retrieval at the execution of all 200 query images for each feature vector is shown in the following tables. 


\begin{tabular}{|c|r|r|r|r|r|r|r|}
\hline & \multicolumn{6}{|c|}{ Table 1. CG : 64 BINS ED PRCP : MEAN } \\
\cline { 2 - 8 } & & & & & \multicolumn{2}{|c|}{ LIN EQUATION123 } \\
\hline MOMENT & ORG & EQH & POLY & LOG & LINEQ1 & LINEQ2 & LINEQ3 \\
\hline R & 5103 & 4644 & 5198 & 5040 & 5098 & 4994 & 4991 \\
\hline G & 5747 & 4933 & 6061 & 5848 & 5960 & 5871 & 5885 \\
\hline B & 5578 & 5363 & 5832 & 5781 & 5865 & 5763 & 5779 \\
\hline
\end{tabular}

\begin{tabular}{|c|c|c|r|r|r|r|r|}
\hline & \multicolumn{7}{|c|}{ Table 2. CG : 64 BINS AD PRCP : MEAN } \\
\cline { 2 - 8 } & & & & & \multicolumn{1}{c|}{ LIN EQUATION123 } \\
\cline { 5 - 8 } MOMENT & ORG & EQH & POLY & LOG & LINEQ1 & LINEQ2 & LINEQ3 \\
\hline R & 6433 & 5264 & 6688 & 6414 & 6521 & 6554 & 6457 \\
\hline G & 6520 & 5525 & $\mathbf{6 7 9 6}$ & 6606 & 6689 & 6653 & 6694 \\
\hline B & 6343 & 5857 & 6517 & 6486 & 6625 & 6505 & 6529 \\
\hline
\end{tabular}

\begin{tabular}{|c|c|r|r|r|r|r|r|}
\hline & \multicolumn{7}{|c|}{ Table 3. CG : 64 BINS CD PRCP : MEAN } \\
\cline { 2 - 8 } & & & & & \multicolumn{1}{c|}{ LIN EQUATON123 } \\
\cline { 3 - 8 } MOMENT & ORG & EQH & POLY & LOG & LINEQ1 & LINEQ2 & LINEQ3 \\
\hline R & 4712 & 4583 & 4953 & 4722 & 4595 & 4591 & 4613 \\
\hline G & 5549 & 4955 & 5953 & 5763 & 5689 & 5642 & 5685 \\
\hline B & 5410 & 5284 & 5805 & 5621 & 5521 & 5443 & 5497 \\
\hline
\end{tabular}

6.2.1. PRCP: In first three tables we are displaying the results obtained for parameter PRCP with ED, AD and CD measures respectively for first moment MEAN. Each table contains the PRCP results for approaches based on four modification functions EQH, POLY, LOG and LinearEQ123 along with the original histogram based bins. These tables are the results obtained by considering the each color feature vector R, G and B separately. Each entry in the table is total retrieval for 200 images i.e., out of 20,000; as for each query it is out of 100 and so for 200 queries it is out of 20,000.

We can observe the above results and can say that, minimum PRCP obtained is 4591 for linear equation 2 in Table 3. The maximum among all is 6796. These results as per the expectation from the ideal system point of view are found very less. Same set of results are obtained for other moments with all other modification functions and we found them reached to maximum value of PRCP as 7259 for Kurtosis moment with polynomial modification function with blue color and AD measure. When we observed all these results for each color R, G and B separately we found them less. To improve the retrieval in terms of PRCP we thought of combining the R, G and B results using OR criterion so that best from each color can be taken into final retrieval. This OR criterion is applied over all sets of results obtained for 200 query images. The following Tables 4, 5 and 6 are showing the results after applying OR operation over R, G, B color results of all other factors (moments, modification functions etc) with ED, AD and CD measure respectively. We found these results far better than separate retrieval based on R, G and B colors. In Table 4, 5 and 6 each entry corresponds to total retrieval (PRCP) out 
of 20,000 i.e., for total PRCP for 200 query images. Each table is highlighting the best results for each moment presented in each row separately. First thing we observed in these tables is that each row when we point out the best results it is actually the comparative analysis of modification functions that which one is performing better for that moment.

According to this observation we can say that in ED measure i.e., Table 4; LinearEQ1, LinearEQ1, POLY and LOG are performing for MEAN, STD, SKEW and KURTO moments respectively. The PRCP obtained is 10142 for STD with LinearEQ1. In table 5 we found LinearEQ1, POLY, LOG and LOG are performing better for first four moments respectively. Similarly in table 6 the best sequence of modification function's performance is POLY, LOG, LOG and LOG for first four moments respectively. On the cumulative analysis of all these results we can say that out of 12 cases of comparisons with respect to four moments and three measures we found that out of 12 at 6 places LOG is performing better among all modifications of histogram. In remaining 6 cases 3 better results are from POLY and 3 from LinearEQ1

\begin{tabular}{|l|c|c|c|c|c|c|c|}
\hline \multirow{2}{*}{ MOMENT } & \multicolumn{6}{|c|}{ Table 4. CG : 64 BINS ED 'R' OR 'G' OR 'B' } \\
\cline { 2 - 8 } & \multirow{2}{*}{ ORG } & \multirow{2}{*}{ EQH } & \multirow{2}{*}{ POLY } & \multirow{2}{*}{ LOG } & \multicolumn{3}{|c|}{ LIN EQUATION123 } \\
\cline { 5 - 8 } & & & & & LINEQ1 & LINEQ2 & LINEQ3 \\
\hline MEAN & 8422 & 7608 & 8649 & 8437 & $\mathbf{8 6 6 1}$ & 8581 & 8608 \\
\hline STD & 9408 & 7626 & 9504 & 9536 & $\underline{\mathbf{1 0 1 4 2}}$ & 10119 & 10071 \\
\hline SKEW & 8990 & 7395 & $\mathbf{9 0 9 4}$ & 9157 & 9042 & 8917 & 8773 \\
\hline KURTO & 9681 & 7782 & 9737 & $\mathbf{9 7 6 8}$ & 9627 & 9486 & 9317 \\
\hline
\end{tabular}

\begin{tabular}{|l|c|c|c|c|c|c|c|}
\hline \multirow{3}{*}{ MOMENT } & \multicolumn{6}{|c|}{ Table 5. CG : 64 BINS AD 'R' OR 'G' OR 'B' } \\
\cline { 2 - 8 } & \multirow{2}{*}{ ORG } & \multirow{2}{*}{ EQH } & \multirow{2}{*}{ POLY } & \multirow{2}{*}{ LOG } & \multicolumn{3}{|c|}{ LIN EQUATION123 } \\
\cline { 5 - 8 } & & & & & LINEQ1 & LINEQ2 & LINEQ3 \\
\hline MEAN & 9418 & 7496 & 9597 & 9422 & $\mathbf{9 6 4 2}$ & 9577 & 9551 \\
\hline STD & 10110 & 8165 & $\mathbf{1 0 3 2 8}$ & 10274 & 9402 & 9252 & 9112 \\
\hline SKEW & 9710 & 7903 & 9899 & $\mathbf{9 9 2 1}$ & 9736 & 9769 & 9704 \\
\hline KURTO & 10225 & 8289 & 10396 & $\underline{\mathbf{1 0 4 1 2}}$ & 10235 & 10340 & 10215 \\
\hline
\end{tabular}

\begin{tabular}{|l|c|c|c|c|c|c|c|}
\hline \multirow{2}{*}{ MOMENT } & \multicolumn{5}{|c|}{ Table 6. CG : 64 BINS CD 'R' OR 'G' OR 'B' } \\
\cline { 2 - 8 } & \multirow{2}{*}{ ORG } & \multirow{2}{*}{ EQH } & \multirow{2}{*}{ POLY } & \multirow{2}{*}{ LOG } & \multicolumn{3}{c|}{ LIN EQUATION123 } \\
\cline { 6 - 8 } & & & & LINEQ1 & LINEQ2 & LINEQ3 \\
\hline MEAN & 7853 & 7536 & $\mathbf{8 3 5 1}$ & 8070 & 8040 & 8041 & 8130 \\
\hline STD & 9243 & 7732 & 9297 & $\mathbf{9 3 5 1}$ & 9232 & 9089 & 8901 \\
\hline SKEW & 8762 & 7500 & 8902 & $\mathbf{8 9 3 7}$ & 8758 & 8729 & 8481 \\
\hline KURTO & 9496 & 7909 & 9471 & $\mathbf{9 5 9 6}$ & 9478 & 9370 & 9119 \\
\hline
\end{tabular}

Analyzing the performance of moments, we found STD and KURTO are better among all four as it is showing the highest retrieval crossing 50\% (10328 and 10412 out of 20,000) 
retrieval of query relevant images from database of 2000 images. Comparing the performances of similarity measures we found $\mathrm{AD}$ is best amongst three of them.

6.2.2. LS: Longest String: This parameter gives the longest continuous string of relevant images. Same set of 200 query images are executed and for each of them LS is calculated. While getting the results of LS we have analyzed the performance of each color separately. At execution of all 10 queries from each class with three different color feature we have taken the maximum LS into consideration and the color of the same is marked. This is done for all classes with all other factors considered in various types of feature vectors with all three similarity measures. Results obtained for longest string are shown in following Chart 1 to Chart 4 for Mean, Standard deviation, Skewness and Kurtosis respectively. As discussed earlier we have taken the maximum LS into consideration. Along with the maximum LS we have also calculated the average LS for those 20 queries; one query (with max result) from each of the 20 classes.

Chart 1. LS: MEAN with ED, AD and CD

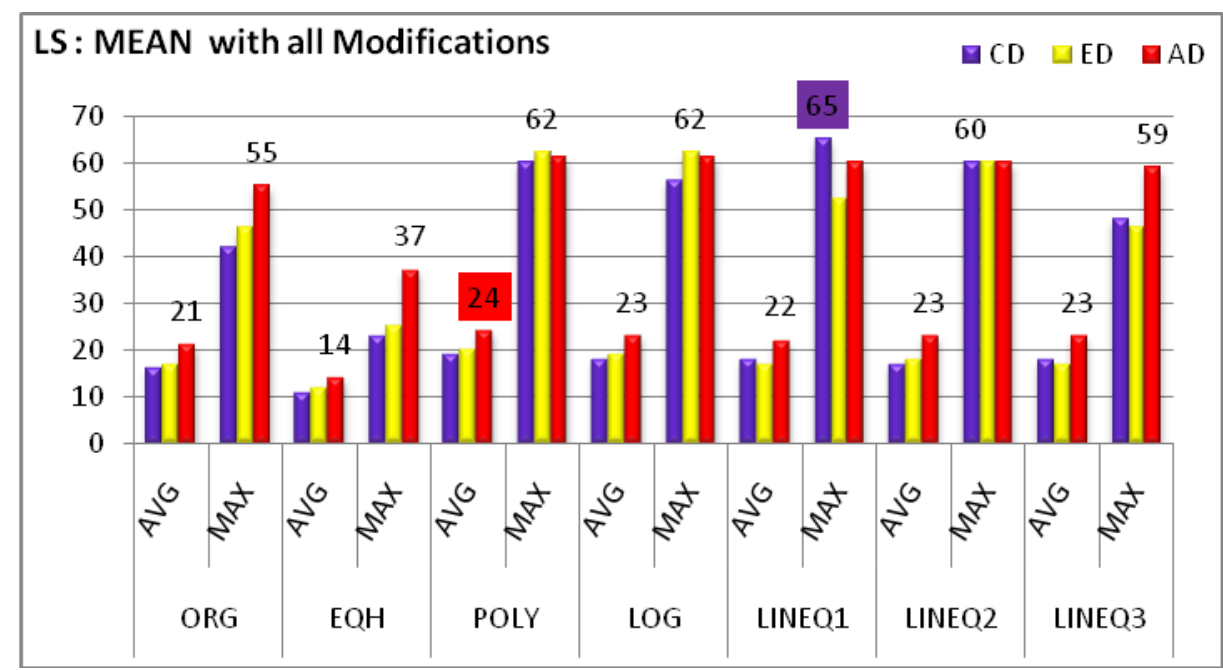

Chart 2. LS: STD with CD, ED and AD

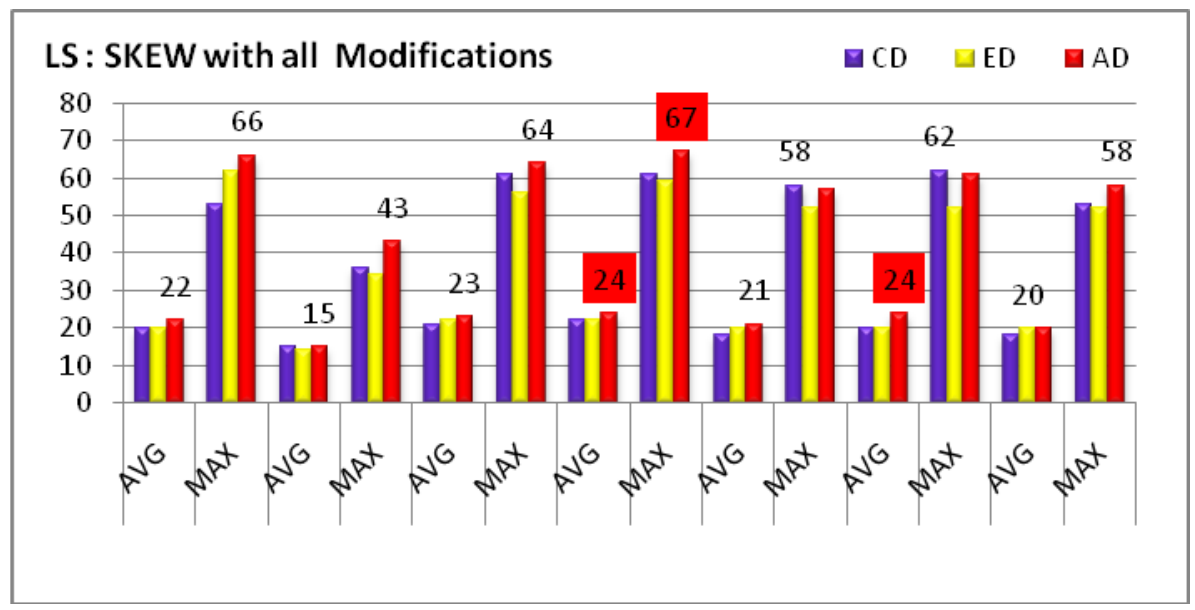


Chart 3. LS: STD with CD, ED and AD

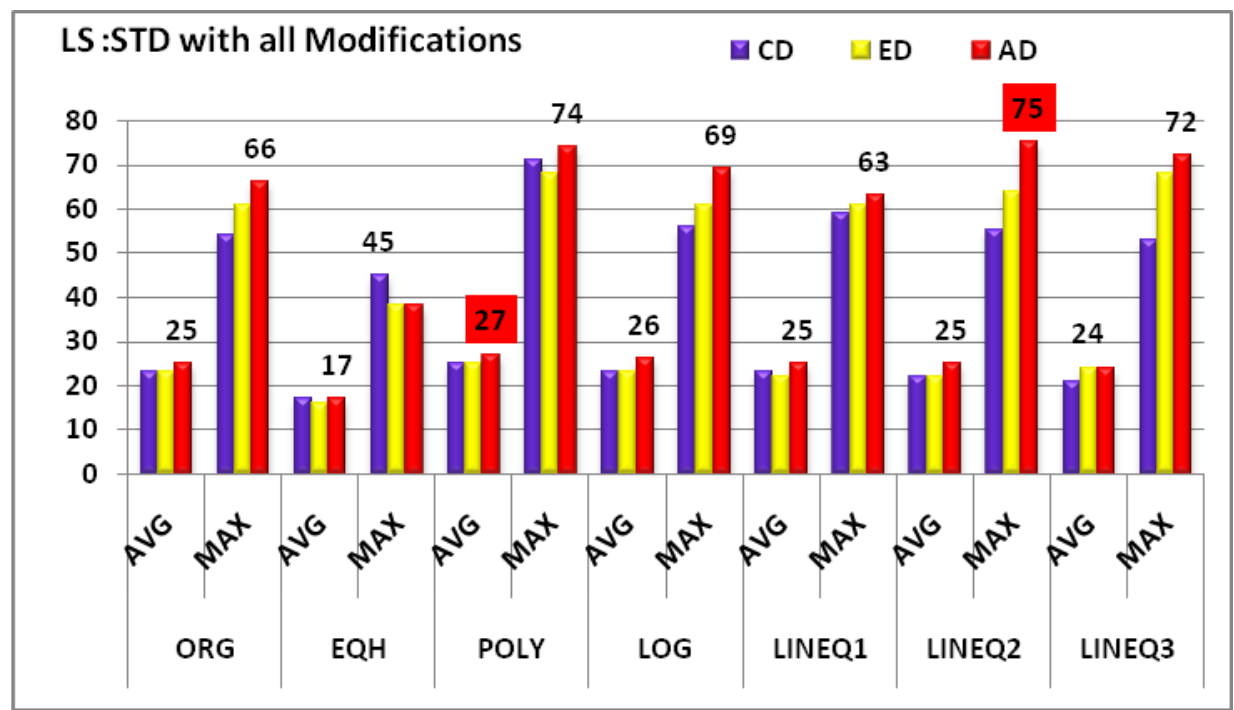

Chart 4. LS: KURTO with CD, ED and ED

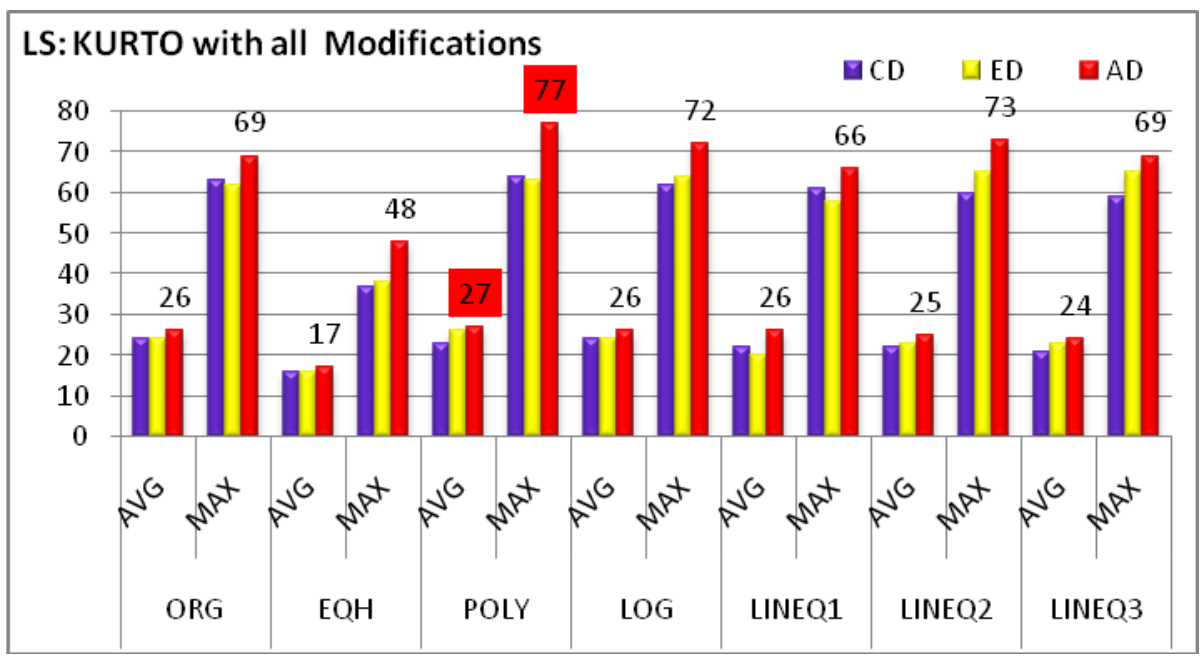

In the above Charts 1 to 4, there are two column sets for each type of histogram (original and the modified ones) i.e., AVG and MAX. Observing these four charts we found that at almost all cases the average of 20 classes crossing 20 and the maximum LS values are crossing 40 which is indicating quite good performance of the proposed approaches. In Charts 1 to 4 each best i.e., AVG and MAX results are highlighted with respective color of similarity measure. It also tells us that the maximum best results are obtained from $\mathrm{AD}$ measure. $\mathrm{CD}$ is also found be good where $\mathrm{ED}$ and $\mathrm{AD}$ are low. While talking about the analysis of performance of the three colors we found for original histogram based results green is better, for function EQH blue color is better, for POLY red and green are equally better, for LOG we found green and in linear equation got green as better amongst three. The best results obtained in all longest string results is 77 as maximum LS for Kurtosis parameter with POLY modifications and 27 as average $L S$ of 20 queries 
6.2.3. LSRR: Length of string to retrieve all relevant images is the simple definition of LSRR. It gives us the length of the path or string of all database images arranged in ascending order to be travelled to collect all images relevant to query where recall will become 1 . Similar to LS; same process is followed while executing the 200 queries for LSRR. However in LS we are taking only MAX LS among 10 queries of each class, but for LSRR we take only minimum values. Following charts 5 to 8 are showing the results obtained for LSRR with all modification function with three similarity measures for MEAN, STD, SKEW and KURTO respectively. $\mathrm{Y}$ axis shows the average and minimum distance to be traversed i.e., LSRR in \% traversal (of string of 2000 images) and X axis shows the modification functions with AVG and MIN results of LSRR. The best values are highlighted with the data labels. The minimum and Average minimum of 20 queries are highlighted with respective color of similarity measure. The color analysis for LSRR is similar to LS. The best LSRR values obtained for LSRR is 7\% means 7\% traversal of 2000 images i.e., till 140th image system will retrieve all query relevant images from the database. The average best performance is $52 \%$ as shown in the charts. One more observation regarding LSRR is that all traversals are below 70\% i.e., sign of better performance of the system.

\section{Chart 5. LSRR: MEAN with ED, AD and CD}

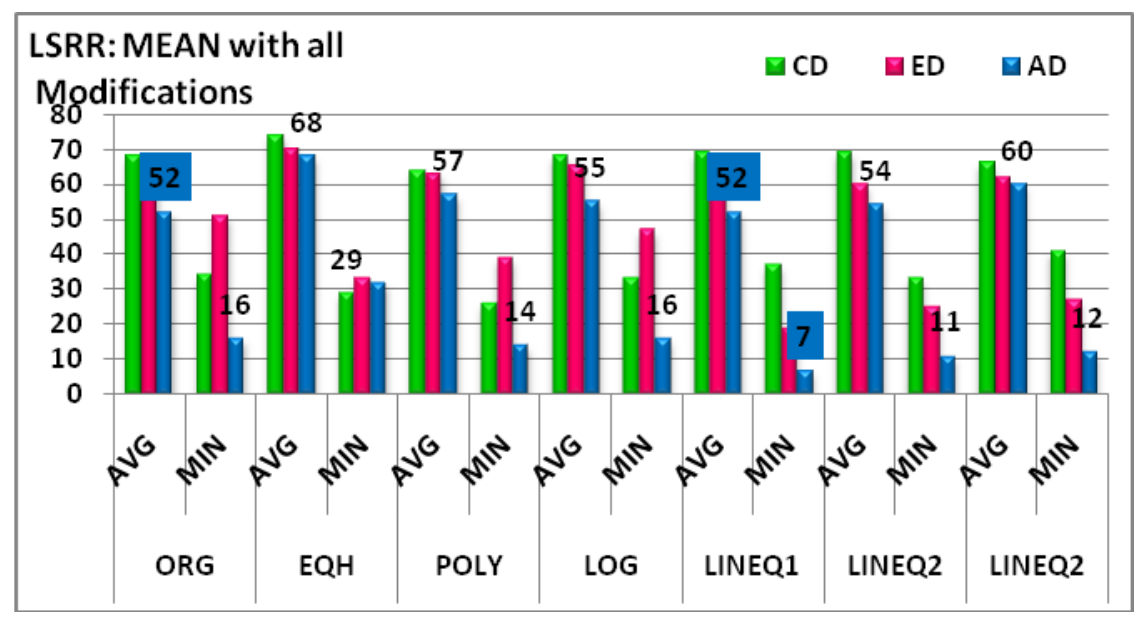

Chart 6. LSRR: STD with ED, AD and CD

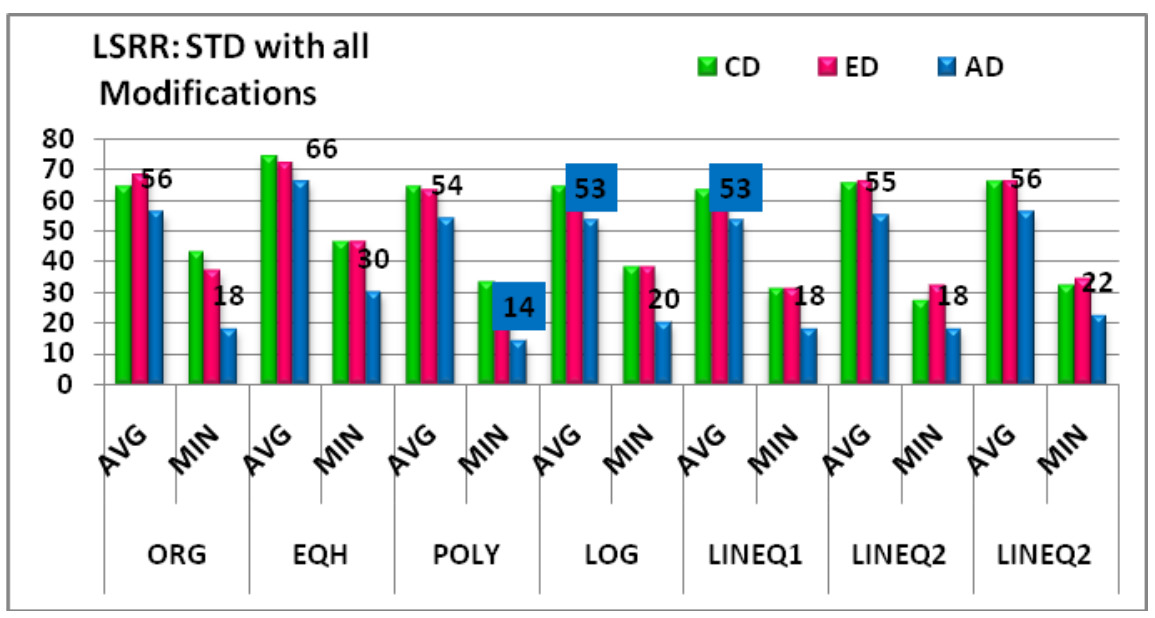


Chart 7. LSRR: SKEW with ED, AD and CD

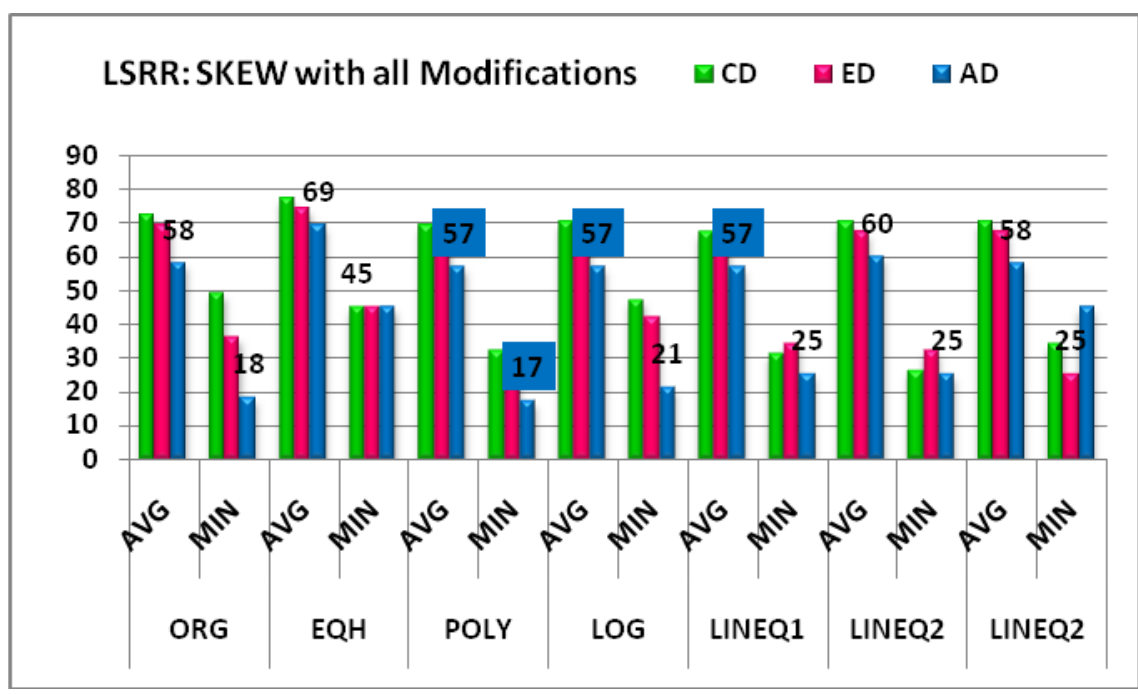

Chart 8. LSRR: KURTO with ED, AD and CD

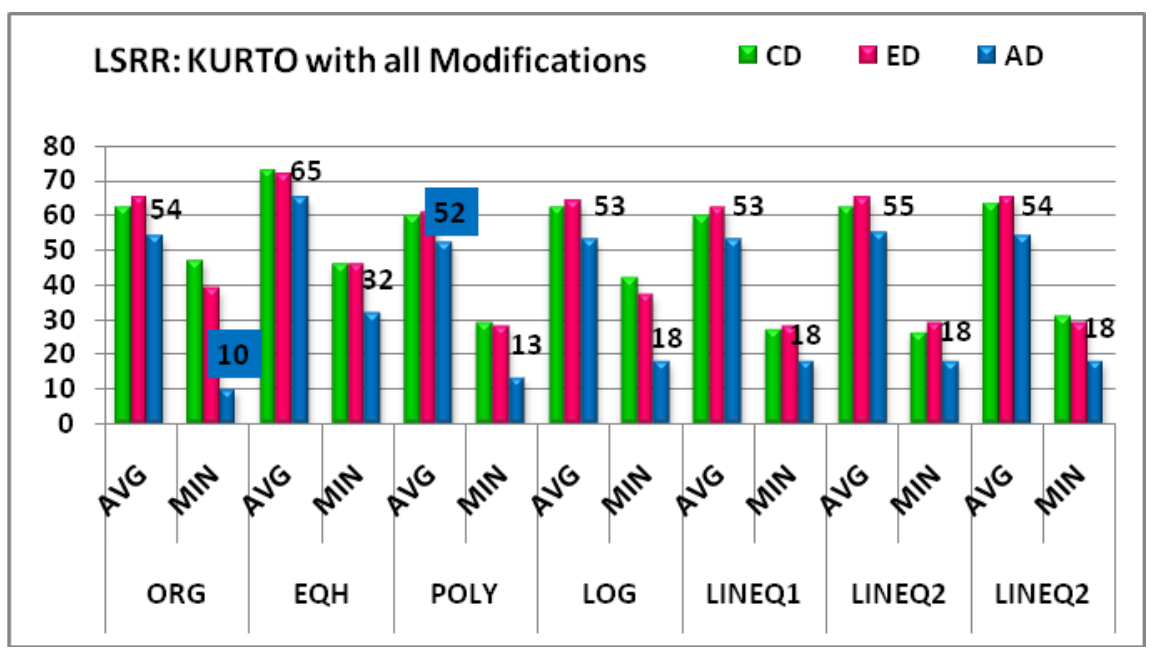

\section{Conclusion}

The Proposed approaches designed for content based image retrieval system are based on histogram partitioning based bins concept. The Bins concept developed in this paper is reducing the size of the feature vector to just 64 bins. It saves the computational time required for the images to compare. Along with the reduction in the feature vector dimension the significance of the image intensities is also considered and handled separately for three colors $R, G$ and $B$. The computation of statistical moments for each of the $R, G$ and $B$ intensities collected into 64 bins proves the use of texture feature along with color contents of the image for formation of the feature vector.

This work mainly highlights the role of different histogram modification functions compared with the original histogram based method. The idea behind application of these modification functions is to enhance the image contents so that feature vector formed using 
them will have good discriminating power to represent that image. Different functions used for modification are EQH, POLY, LOG and Linear EQ1, 2, \& 3. Among these four functions LOG and POLY are found to be best for PRCP parameter. The PRCP values are crossing 0.5 which are showing the achievement of quite better results in CBIR field. Overall observation of PRCP indicates that all modifications are performing better as compared to original as well as Equalized histogram which is actually found most commonly used histogram specification in CBIR [27-32, 34-36].

Performance evaluated using the LS and LSRR also proved the system's potential to retrieve the similar images. In LS, the value should be as high as possible and here the best values obtained with consideration of all approaches are reaching to 75 in many of the queries. However the other parameter LSRR, the ideal value should be as low as possible. Because the minimum is the distance to be traversed the less time system takes to collect all relevant images from database which is always desired by all CBIR users. The best values obtained here are about just 10 to $15 \%$ which is quite good achievement to fulfill the CBIR user's expectations.

Time consumed by the system for the retrieval depends on dimension of the feature vector and also on the similarity measure used for comparing the images. Here three similarity measures are used namely $\mathrm{CD}, \mathrm{ED}$ and $\mathrm{AD}$. Among them we found $\mathrm{AD}$ is best which has very low computational complexity; whereas $\mathrm{CD}$ and $\mathrm{ED}$ are eventually performing well when remaining two measures are fail to perform.

\section{References}

[1] F. Long, H. Zhang and D. Dagan Feng, "Fundamentals of Content-Based Image Retrieval”, Multimedia Information Retrieval andManagement-Technological Fundamentals and Applications, Springer-Verlag, (2003), pp. 1-26.

[2] Y. Rui and T. S. Huang, "Image Retrieval: Current Techniques, Promising Directions, and Open Issues", Journal of Visual Communication and Image Representation, vol. 10, (1999), pp. 39-62.

[3] A. M. W. Smeulders, M. Worring, S. Santini, A. Gupta and R. Jain, "Content-based image retrieval at the end of the early years”, IEEE Transactions On Pattern Analysis and Machine Intelligence, vol. 22, no. 12, pp. 1349-1380, (2000).

[4] R. C. Veltkamp and M. Tanase, "Content-Based Image Retrieval Systems: A Survey”, Technical Report UUCS-2000-34, Dept. Of Computing Science, Utrecht University, [Online]. http://www.aalab.cs.uu.nl/cbirsurvey, (2002).

[5] H. B. Kekre and K. Sonawane, "Retrieval of Images Using DCT and DCT Wavelet Over Image Blocks", (IJACSA) International Journal of Advanced Computer Science and Applications, vol. 2, no. 10, (2011).

[6] H. B. Kekre and K. Sonawane, "Query based Image Retrieval using Kekre, DCT and Hybrid wavelet Transform over 1st and 2nd Moment”, International Journal of Computer Applications (0975 - 8887), vol. 32, no. 4, (2011) October.

[7] H. B. Kekre and K. Sonawane, "Row, Column and Fused Row-Col R, G, B Plane’s Feature Vector Generation using DCT, DST and Kekre Wavelet for CBIR”, ICICT, (2012) October, IEEE Explorer.

[8] P. S. Suhasini and K. Sri Rama Krishna, “CBIR Using Color Histogram Processing”, Journal of Theoretical and Applied Information Technology, www.jatit.org, (C) (2005-2009).

[9] G. Jaswal, A. Kaul and R. Parmar, "Content based Image Retrieval using Color Space Approaches”, International Journal of Engineering and Advanced Technology (IJEAT) ISSN: 2249 - 8958, vol. 2, no. 1, (2012).

[10] Y. Deng and B. S. Manjunath, “An Efficicent Low Dimensional Color Indexing Scheme For Region Based Image Retreival”, 0-7803-5041-3/99\$10.00 (C) 1999, IEEE 301.

[11] H. B. Kekre and K. Sonawane, "Standard Deviation of Mean and Variance of Rows and Columns of Images for CBIR”, Published in IJCISSE. http://www.waset.org/ijecse/v1.html, (2009) January.

[12] H. B. Kekre and K. Sonawane, "Feature Extraction in Bins Using Global and Local thresholding of Images for CBIR”, in. IJCISSE, vol. 3, no. 1, winter (2009), pp.1- 4.

[13] X. Li, "Image retrieval based on perceptive weighted color blocks”, Pattern Recognition Letters, vol. 24, (2003), pp. 1935-1941, www.elsevier.com/locate/patrec. 
[14] H. B. Kekre and S. D. Thepade, "Image Retrieval using Color-Texture Features Extracted from Walshlet Pyramid”, ICGST - GVIP Journal, ISSN: 1687-398X, vol. 10, no. 1, (2010) February.

[15] H. B. Kekre and K. Sonawane, "Linear Equation in Parts as Histogram Specification for CBIR Using Bins Approach”, International Journal of Engineering Research and Development e-ISSN: 2278-067X, p-ISSN: 2278-800X, www.ijerd.com, vol. 4, no. 4, (2012) October, pp. 73-85.

[16] H. B. Kekre and K. Sonawane, "Bins Approach To Image Retrieval Using Statistical Parameters Based on Histogram Partitioning of R, G, B Planes”, International Journal of Advances in Engineering \& Technology, CIJAET ISSN: 2231-1963, vol. 2, no. 1, January (2012), pp. 649-659.

[17] H. B. Kekre and K. Sonawane, "Performance improvement using average query fired to bins of four statistical moments for CBIR”, IEEE Explore, (ICCICT), 2012 International Conference, Mumbai, (2012), October 19-20.

[18] S. Wang, “A Robust CBIR Approach Using Local Color Histograms”, Technical Report TR, (2001) October 1-13.

[19] A. Mohamed, F. Khellfi, Y. Weng and J. Jiang, "Stan.Ipson: An efficient Image Retrieval through DCT Histogram Quantization”, 2009 International Conference on CyberWorlds, (2009).

[20] W.-M. Zheng, Z.-M. Lu and H. Burkhardt, "Color Image Retrieval Schemes Using Index Histograms Based On Various Spatial-Domain Vector Quantizers”, International Journal of Innovative Computing, Information and Control ICIC International C 2006 ISSN 1349-4198, vol. 2, no. 6, December (2006).

[21] J. Hafner, H. S. Sawhney, W. Equits, M. Flickner and Wayne Niblack, "Efficient Color Histogram Indexing for Quadratic Form Distance Functions”, IEEE Trans. on Pattern Analysis and Machine Intelligence, vol. 17, no. 7, (1995) July.

[22] G. Pass Ramin Zabih, "Histogram Refinement for Content-Based Image Retrieval”, http://www.cs.cornell.edu/home/rdz/refinement.html, 0-8186-7620 1996 IEEE.

[23] G.-H. Yoo and B. Kyun Kim, "Content-Based Image Retrieval Using Shifted Histogram”, ICCS 2007, Part III, LNCS 4489, 2007 (C) Springer-Verlag Berlin Heidelberg, (2007), pp. 894-897.

[24] D. Zhang and G. Lu, "Evaluation of similarity measurement for image retrieval”, IEEE International Conf. on Neural Networks Signal, (2003), pp. 928-931.

[25] R. Hu, S. R"uger and H. Di Song, "Dissimilarity Measures For Content-Based Image Retrieval”.

[26] H. B. Kekre and K. Sonawane, "Effect of Similarity Measures for CBIR using Bins Approach”, IJIP, vol. 6, no. 3, (2012).

[27] M. Islam, D. Zhang and G. Lu, "Comparison of Retrieval Effectiveness of Different Region Based Image Representations”, ICICS 2007, 1-4244-0983-7/07/\$25.00 @2007 IEEE.

[28] W. Pedrycz and A. Amato, "Fuzzy Clustering With Partial Supervision in Organization and Classification of Digital Images”, IEEE Transactions On Fuzzy Systems, vol. 16, no. 4, (2008) August.

[29] J. Vogela and B. Schieleb, "Performance evaluation and optimization for content-based image retrieval", 0031-3203/\$30.00, 2005 Pattern Recognition Society. Published by Elsevier Ltd.

[30] Y. Wan, Member, IEEE, and D. Shi, “Joint Exact Histogram Specification and Image Enhancement Through the Wavelet Transform”, IEEE Transactions on Image Processing, vol. 16, no. 9, (2007) September.

[31] S. G. Shaila and A. Vadivel, "Content-Based Image Retrieval Using Modified Human Colour Perception Histogram”, ITCS, SIP, JSE-2012, CS \& IT 04, 2012.@ CS \& IT-CSCP 2012, pp. 229-237.

[32] M. Liu Yanxiang He Huijun Hu, "Dimension Reduction Based on Rough Set in Image Mining”, Proceedings of the Fourth International Conference on Computer and Information Technology (CIT’04) 0-7695-22165/04 \$20.00 (C) 2004 IEEE.

[33] http://wang.ist.psu.edu/docs/related.shtml.

[34] NST Sai and R. C. Patil, “Image Retrieval using Equalized HistogramImage Bins Moments”, IJRTET, vol. 3, no. 1 , (2010).

[35] V. Vijaya Kuma and N. GnaneswaraRao, "IHBM: Integrated Histogram Bin Matching For Similarity Measures of Color Image Retrieval”, International Journal of Signal Processing, Image Processing and Pattern Recognition, vol. 2, no. 3, (2009) September.

[36] M. Braveen and P. Dhavachelvan, "Evaluation of Content Based Image Retrieval Systems Based on Color Feature”, IJRTE, vol. 1, no. 2, (2009) May. 


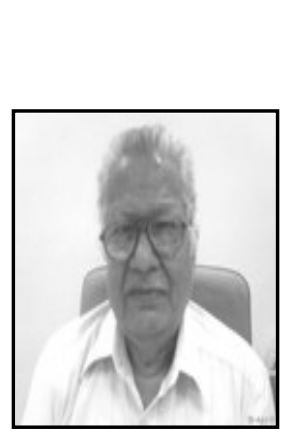

\section{Authors}

H. B. Kekre has received B.E. (Hons.) in Telecomm. Engg. from Jabalpur University in 1958,M.Tech (Industrial Electronics) from IIT Bombay in 1960, M.S. Engg. (Electrical Engg.) from University of Ottawa in 1965 and Ph.D. (System Identification) from IIT Bombay in 1970. He has worked Over 35 years as Faculty of Electrical Engineering and then HOD Computer Science and Engg. at IIT Bombay. For last 13 years worked as a Professor in Department of Computer Engg. at Thadomal Shahani Engineering College, Mumbai. He is currently Senior Professor working with Mukesh Patel School of Technology Management and Engineering, SVKM's NMIMS University, Vile Parle(w), Mumbai, INDIA. He has guided 17 Ph.D.s, 150 M.E./M.Tech Projects and several B.E./B.Tech Projects. His areas of interest are Digital Signal processing, Image Processing and Computer Networks. He has more than 450 papers in National / International Conferences / Journals to his credit. Recently twelve students working under his guidance have received best paper awards. Five of his students have been awarded Ph. D. of NMIMS University. Currently he is guiding eight Ph.D. students. He is member of ISTE and IETE.

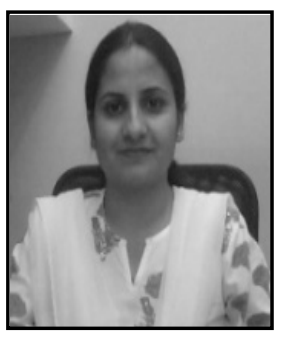

Ms. Kavita V. Sonawane has received M.E (Computer Engineering) degree from Mumbai University in 2008. Pursuing Ph.D. from Mukesh Patel School of Technology, Management and Engg, SVKM's NMIMS University, Vile-Parle (w), Mumbai, INDIA. She has more than 9 years of experience in teaching. Currently working as a Assistant professor in Department of Computer Engineering at St. Francis Institute of Technology Mumbai. Her area of interest is Image Processing, Data structures and Computer Architecture. She has 23 papers in National/ International conferences / Journals to her credit. She is member of ISTE. 